\title{
CURVES IN CAGES: AN ALGEBRO-GEOMETRIC ZOO
}

\author{
GABRIEL KATZ
}

\section{Introduction.}

An algebraic plane curve is the solution set of a polynomial equation $P(x, y)=0$, where $x$ and $y$ are real or complex variables. By definition, the degree of the curve is the degree of the polynomial $P(x, y) .{ }^{1}$ When $P(x, y)$ is a product of two non-constant polynomials over a given number field, the curve is called reducible; otherwise, it is irreducible.

This paper is concerned with families of plane algebraic curves that contain a given and quite special set of points $\mathcal{X}$. We focus on the case in which the set $\mathcal{X}$ is formed by transversally intersecting pairs of lines selected from two given finite families. The union of all lines from both families is called a cage (this notion of cage will be made more precise later), and the intersection $\mathcal{X}$ consists of points at which a line from the first family intersects a line from the second. The points of $\mathcal{X}$ are called the nodes of the cage.

This is a particular case of a more general problem. Let $\mathcal{X}$ be the intersection set of two plane algebraic curves $\mathcal{D}$ and $\mathcal{E}$ that do not share a common component, that is, do not contain a common irreducible curve. If $d$ and $e$ denote the degrees of $\mathcal{D}$ and $\mathcal{E}$, respectively, then $\mathcal{X}$ consists of at most $d \cdot e$ points. When the cardinality of $\mathcal{X}$ is exactly $d \cdot e, \mathcal{X}$ is called a complete intersection (complete intersections have many nice properties). How does one describe polynomials of degree at most $k$ that vanish on a complete intersection $\mathcal{X}$ or on its subsets? This problem has a glorious history (see $[\mathbf{1}],[\mathbf{2}]$, or $[\mathbf{3}]$ ) and its generalizations are a subject of active and exciting research $[\mathbf{4}],[\mathbf{5}],[\mathbf{6}],[\mathbf{7}],[\mathbf{8}],[\mathbf{9}]$. When $k$ is much larger then $d \cdot e$, points of $\mathcal{X}$ impose independent restrictions on polynomials of degree $k$. However, for small $k$ these restrictions fail to be independent. In such cases, we can look for maximal subsets of $\mathcal{X}$ that impose independent constraints.

In this article, we deal with the special case of this classical problem suggested earlier, namely, the case in which both plane curves $\mathcal{D}$ and $\mathcal{E}$ are simply unions of lines and the union $\mathcal{D} \cup \mathcal{E}$ is the $(d \times e)$-cage in question. Note that $\mathcal{D}$ is the zero set of a product of $d$ linear polynomials, while $\mathcal{E}$ is the zero set of a product of $e$ linear polynomials. Hence, the degrees of $\mathcal{D}$ and $\mathcal{E}$ are $d$ and $e$, respectively. In order to simplify our terminology, we color the lines from $\mathcal{D}$ red and the ones from $\mathcal{E}$ blue.

The case of cages is amenable to elementary methods that presume only a modest familiarity with algebraic geometry. Moreover, its beautiful geometric applications are the true focus of our exposition. These applications can be viewed as natural

\footnotetext{
${ }^{1}$ According to this point of view adapted by algebraic geometers, the degree is associated with the equation rather than with its solution set: for example, the equation $\left(x^{2}+y^{2}-1\right)^{2}=0$ defines a "double circle"" of degree four.
} 
generalizations of classical theorems in the foundations of projective geometry. The reader familiar with Pascal's theorem can get a feel for the nature of these generalizations by glancing at Figures 6 and 7 and comparing them with the classical Pascal diagram in Figure 1.

The zoo of algebraic curves attached to the nodes of a cage is a microcosm of classical algebraic geometry. We invite the Monthly readership to join us on a tour of its feature attractions.

My interest in the subject was sparked by N. B. Vasiliev's engaging paper "Pascal's Hexagrams and Cubic Curves" [13]. The striking and well-known connection between classical theorems of projective geometry and the algebraic geometry of cubic plane curves was a revelation to me. Equally striking was the elegance of the argument that established this connection. This article was written in the afterglow of this private epiphany.

\section{Pascal's Mystic Hexagram.}

In 1640, a the sixteen-year-old Blaise Pascal discovered a remarkable property of a hexagon inscribed in a circle. In a diagram generated by the hexagon, three specific intersection points always happen to be collinear! He called the configuration " The Mystic Hexagram," made fifty posters of it, and mailed them to fellow scientists.

Shortly thereafter, Pascal realized that a similar observation holds for a hexagon inscribed in an ellipse. In fact, the amazing collinearity is preserved under central and parallel projections of his diagram (see Figure 1): a projection maps lines to lines, the circle is transformed into a quadratic curve, and the inscribed hexagon is mapped onto a hexagon inscribed in that curve.

Pascal's mystic hexagram was a fundamental result in geometry unknown to the classical Greek school. In the words of Fermat, "we learned that the ancient Greeks did not know everything about geometry." Along with Desargues's theorem (discovered four years earlier), Pascal's theorem (see [11, Corollary 3.15]) gave birth to a new branch of nonmetrical geometry that we now call projective geometry.

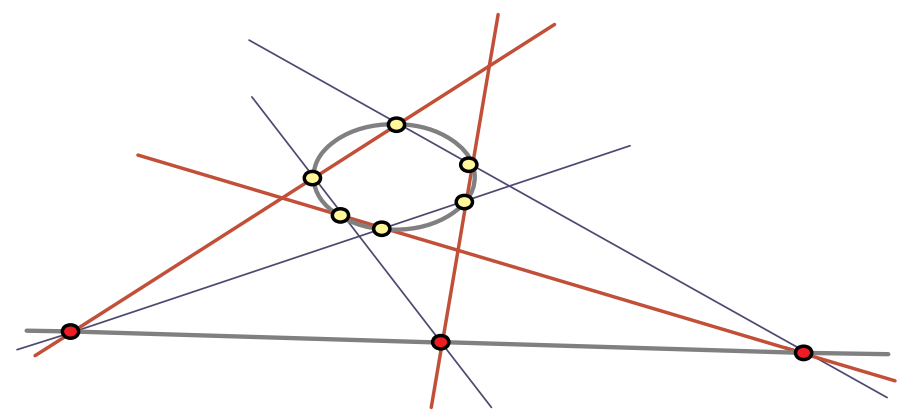

Figure 1. Pascal's mystic hexagram. 
Theorem 2.1. (Pascal's Mystic Hexagram). Given a hexagon inscribed in a quadratic curve $\mathcal{Q}$, color its edges alternately blue and red and extend them. Generically, the resulting three red lines intersect the three blue lines at three points different from the six vertices of the hexagon. These three points must be collinear.

The three blue and three red lines generated by the hexagon form a $(3 \times 3)$-cage. In general, two triples of lines produce nine nodes (intersection points), each of which belongs to a unique pair of one red line and one blue line.

In the mystic hexagram, six of the nine nodes lie on the quadratic curve $\mathcal{Q}$, while the remaining nodes (by the Pascal theorem) must lie on a line $\mathcal{L}$. Consider the union $\mathcal{C}=\mathcal{Q} \cup \mathcal{L}$. The set $\mathcal{C}$ is an example of a reducible cubic curve - it is the zero set of the product of two polynomials in two variables, one quadratic and one linear.

We can now formulate a well-known corollary of Pascal's theorem:

Theorem 2.2. All nine nodes of the $(3 \times 3)$-cage generated by a hexagon inscribed in a quadratic curve $\mathcal{Q}$ lie on a reducible cubic curve $\mathcal{C}=\mathcal{Q} \cup \mathcal{L}$.

Of course, the line $\mathcal{L}$ is determined by any pair $a$ and $b$ from the three new nodes $a, b$, and $c$ of the cage. Thus, eight nodes of the cage - the six vertices of the inscribed hexagon and the new pair $\{a, b\}$-always belong to some cubic curve $\mathcal{C}$. What happens to the remaining ninth node $c$ ? According to the Pascal theorem, the ninth node must lie on $\mathcal{C}$ as well! This reformulation of Pascal's theorem leads one to wonder whether the theorem might be a general cubic phenomenon. Might it hold for a hexagon inscribed in a general cubic curve? Perhaps the reducibility of $\mathcal{C}=\mathcal{Q} \cup \mathcal{L}$ is merely an accident. The following classical proposition (see $[\mathbf{1 2}$, Corollary 2.7]) validates these insights (Figure 2):

Theorem 2.3. (Cage Theorem for Cubics). Any cubic curve $\mathcal{C}$ that passes through eight nodes of a $(3 \times 3)$-cage must pass through the ninth node.

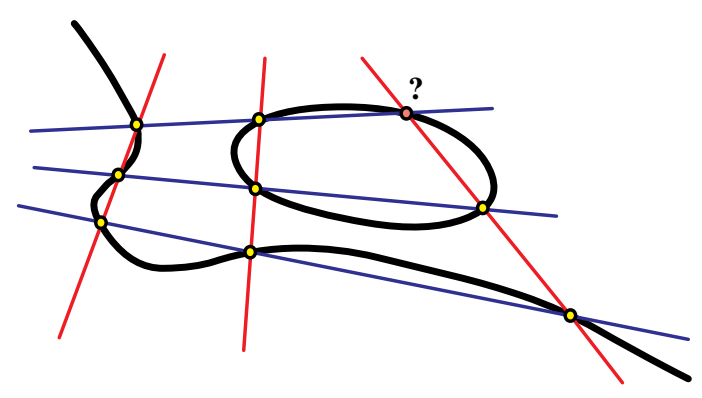

FiguRE 2. The cage theorem for cubics.

A natural generalization of Theorem 2.3 is a theorem of Chasles [3]. It claims that if $\mathcal{X}$ is a complete intersection of two cubic curves, then any cubic curve that contains eight points from $\mathcal{X}$ will include its ninth point as well.

Before proceeding, we issue one caveat to the reader. Although the diagrams in this paper suggest the case of real algebraic curves, all our algebraic arguments and the results make sense - and are even simpler - for complex algebraic curves (defined by complex polynomials). 
At first glance, Theorem 2.3 appears to describe an esoteric fact. However, it reflects a wonderful intrinsic structure shared by nonsingular cubic curves (called elliptic curves). It turns out that any elliptic $\mathcal{C}$ curve has the structure of an Abelian group (see $[\mathbf{1 1}, 3.14]$ ).

We recall for the reader how the group operation $(x, y) \longmapsto x+y$ is defined for an elliptic curve $\mathcal{C}$. Here we view $\mathcal{C}$ as residing in a projective plane (see section 3 for a short discussion of projective curves and spaces). If $x$ and $y$ are distinct points of $\mathcal{C}$, then there is a unique point $z$ of $\mathcal{C}$ with the property that $x, y$, and $z$ are collinear; if $x=y$, then the tangent line to $\mathcal{C}$ at $x$ hits the curve at a single point $z$. Next, we designate one point on $\mathcal{C}$ as the additive identity element $e$. Once $e$ is chosen, $z$ must play the role of $-(x+y)$. In other words, $x+y$ is uniquely determined by the property: $x+y, z$, and $e$ are collinear points. Evidently, for each $x$ in $\mathcal{C}$ we have $x+e=x$. When there is one group structure on a set, there are of course many others obtained from the given structure by conjugation with a translation. A translation sends the identity element $e$ to another element, which becomes the identity element of the new structure. It is customary to pick one of the inflection points of $\mathcal{C}$ for the role of $e$.

In the language of the group structure on an elliptic curve, the cage theorem becomes a statement about the associativity of the binary group operation! It's a subtle interpretation. Figure 3 indicates how it works.

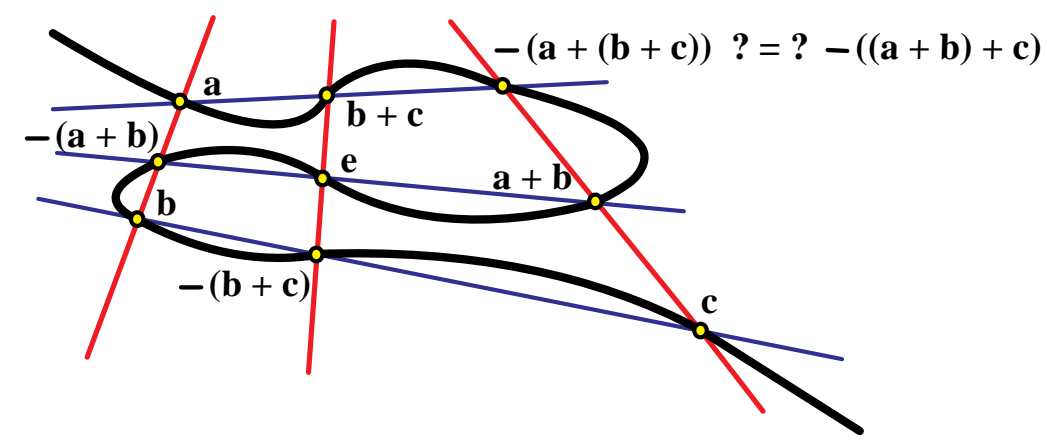

FiguRE 3.

It is striking to realize that every classical theorem of planar projective geometry is, in a sense, an implication of Theorem 2.3 for cubics, which in turn reflects the associativity of point addition on nonsingular cubic curves! So elliptic curves and planar projective geometry are intimately related.

One might wonder whether a different, more direct generalization of Pascal's theorem is valid: Is it true that any alternately-colored hexagon inscribed in a cubic curve produces a $(3 \times 3)$-cage all of whose nodes lie on the curve? A simple count of dimensions rules out this possibility. For any cubic curve $\mathcal{C}$ the variety of all hexagons inscribed in $\mathcal{C}$ is six-dimensional. One can show that the family of hexagons whose $(3 \times 3)$-cages have all their nodes on $\mathcal{C}$ constitutes a four-dimensional variety. $^{2}$ Thus, for an arbitrary $\mathcal{C}$, not any inscribed hexagon will do - it is important for $\mathcal{C}$ to have a quadratic component $\mathcal{Q}$ in which the hexagons can be inscribed!

\footnotetext{
${ }^{2}$ By Theorem 2.3, a generic quadrilateral inscribed in $\mathcal{C}$ gives rise to a unique $(3 \times 3)$-cage whose nodes belong to $\mathcal{C}$.
} 
In particular, not any hexagon inscribed in a union of a quadratic curve with a line will generate a cage that belongs to that union.

\section{HIGH DEGREE CURVES IN CAGES.}

We aim in this article to generalize some of the observations made in the previous section for cubic curves to curves of any degree $d$ that pass through the nodes of a $(d \times e)$-cage, where $e \leq d$. Most of our results can be derived from the Bacharach duality theorem [1], [5] (see also Theorem 4.1, as outlined in section 4). However, our goal here is to replace the powerful machinery of algebraic geometry with more elementary considerations that rely only on the divisibility of polynomials.

Our arguments are identical for real or complex curves, meaning for curves over a ground field $\mathbb{A}$ with either $\mathbb{A}=\mathbb{R}$ or $\mathbb{A}=\mathbb{C}$. They apply both to curves in the affine $x y$-plane $\mathbb{A}^{2}$ and to curves in the projective plane $\mathbb{P}^{2}$ with homogeneous coordinates $[x: y: z]$. Recall that the points of $\mathbb{P}^{2}$ are the proportionality classes of triples $(x, y, z)$ different from $(0,0,0)$ (i.e., the equivalence classes $[x: y: z]$ of $\mathbb{A}^{3} \backslash\{(0,0,0)\}$ modulo the relation " $\sim$ ": $(x, y, z) \sim(\lambda x, \lambda y, \lambda z)$ for any $(x, y, z)$ in $\mathbb{A}^{3} \backslash\{(0,0,0)\}$ and any $\lambda$ in $\mathbb{A}^{*}$, the multiplicative group of nonzero elements of $\mathbb{A})$. Depending on the context, the numbers $x, y$, and $z$ can be real or complex. For the most part, our notations are neutral with respect to the choice of the ground field $\mathbb{A}$.

The introduction of the projective plane simplifies the intersection theory of algebraic curves. For example, in contrast with the plane $\mathbb{A}^{2}$, in the projective plane $\mathbb{P}^{2}$ any two lines have an intersection point.

To put matters into context, we recall for readers a few basic notions. Each polynomial $P(x, y)$ of degree at most $d$ gives rise to a unique homogeneous polynomial in $x, y$, and $z$ of degree $d$. This is done simply by replacing each monomial $x^{a} y^{b}$ in $P(x, y)$ with the monomial $x^{a} y^{b} z^{d-a-b}$.

An affine (algebraic) curve in $\mathbb{A}^{2}$ is the zero set of a single polynomial in the variables $x$ and $y$, while a projective curve in the projective plane $\mathbb{P}^{2}$ is the zero set of a homogeneous polynomial in $x, y$, and $z$. The degree of a curve is the degree of its defining polynomial. Two polynomials that are proportional define the same curve. Therefore, we interpret the set of proportionality classes of polynomials in $x$ and $y$ of degree $d$ as the set of plane affine curves of degree $d$. Similarly, the set of proportionality classes of homogeneous polynomials in $x, y$, and $z$ of degree $d$ may be seen as the set of projective curves of degree $d$ in $\mathbb{P}^{2}$. For curves over the real numbers this is a simplistic model (many polynomials have empty zero sets), but over the complex numbers it is more than adequate.

A polynomial $P(x, y)$ of degree not larger than $d$ has

$$
1+2+3+\cdots+d=(d+1)(d+2) / 2
$$

coefficients. Such polynomials, taken up to proportionality, constitute an $n$-dimensional projective space $\mathbb{P}_{*}^{n}$, where

$$
n=[(d+1)(d+2) / 2]-1=\left(d^{2}+3 d\right) / 2 .
$$

Its points are the proportionality classes of sequences formed by the coefficients of $P(x, y)$.

When $k<d$, the polynomials in $x$ and $y$ of degree at most $k$ are contained in the set of polynomials of degree at most $d$. This gives rise to a nested family of 
projective subspaces $\left\{\mathbb{P}_{*}^{\left(k^{2}+3 k\right) / 2}\right\}_{0 \leq k \leq d}$. For example, the family

$$
\mathbb{P}_{*}^{0} \subset \mathbb{P}_{*}^{2} \subset \mathbb{P}_{*}^{5} \subset \mathbb{P}_{*}^{9} \subset \mathbb{P}_{*}^{14} \subset \mathbb{P}_{*}^{20}
$$

corresponds to polynomials $P(x, y)$ of degrees 0 and at most $1,2,3,4$, and 5 , respectively. The affine curves of degree $d$ in $\mathbb{A}^{2}$ form an open set

$$
\mathbb{P}_{*}^{\left(d^{2}+3 d\right) / 2} \backslash \mathbb{P}_{*}^{\left[(d-1)^{2}+3(d-1)\right] / 2}
$$

in the space $\mathbb{P}_{*}^{\left(d^{2}+3 d\right) / 2}$. For instance, in this model the cubic curves in $\mathbb{P}^{2}$ are represented by points of $\mathbb{P}_{*}^{9}$, while the cubic curves in $\mathbb{A}^{2}$ are represented by the set $\mathbb{P}_{*}^{9} \backslash \mathbb{P}_{*}^{5}$.

As $d$ increases, the members of the universe of plane curves of degree $d$ that contain all the nodes of a $(d \times d)$-cage become more and more rare. Indeed, the dimension of the variety of plane curves of degree $d$ is a quadratic function of $d$, whereas the dimension of the subvariety of such curves that contain the nodes of a $(d \times d)$-cage grows only linearly in $d$. Still, among the caged curves there are a few interesting beasts. Consider, for instance, the Fermat curve $\mathcal{F}$ given in homogeneous coordinates $[x: y: z]$ by the equation $x^{d}+y^{d}=z^{d}$, or in Cartesian (i.e. affine) coordinates by the equation $x^{d}+y^{d}=1$. We notice that, over the complex numbers, $\mathcal{F}$ is a curve that contains the nodes of a $(d \times d)$-cage. Simply write the curve's equation in the form $\left(x^{d}-1 / 2\right)+\left(y^{d}-1 / 2\right)=0$ or in the form $\prod_{\xi}(x-\xi)+\prod_{\xi^{\prime}}\left(y-\xi^{\prime}\right)=0$, where $\xi$ and $\xi^{\prime}$ run over the $d$ th roots of $1 / 2$. Thus, all the root pairs $\left(\xi, \xi^{\prime}\right)$ form the nodes of the complex Fermat cage.

Our next goal is to describe polynomials $P$ of degree $d$ that vanish at the nodes of a given $(d \times e)$-cage $K$, where $d \geq e$. By forcing a polynomial $P$ to vanish at a given point of a projective plane, we are imposing a single linear restriction on its coefficients. More points produce further restrictions, and could lower the dimension of the vector space formed by such polynomials. If the degrees of polynomials are not bounded from above, distinct points will impose independent constraints. However, when the degrees are bounded, new points often add only redundant constraints.

By way of example, one expects that requiring a polynomial $P(x, y)$ of degree less then or equal to $d$ to vanish at $\left(d^{2}+3 d\right) / 2$ generic points should nail it down (up to proportionality). Since, a $(d \times d)$-cage has $d^{2}$ nodes, some of them must impose redundant restrictions on the coefficients of $P(x, y)$ (provided that $d \geq 3$ ).

The issue is: Which nodes of a $(d \times e)$-cage are redundant, and how can one describe the variety of degree $d$ polynomials vanishing at the nodes? A few combinatorial definitions, linked to the notion of $k$-configurations in [6], will help us to depict the set of redundant nodes. As before, we mark the two sets of lines forming a cage with two colors. Given any ordering of the red lines $\left\{\mathcal{R}_{1}, \mathcal{R}_{2}, \ldots, \mathcal{R}_{d}\right\}$ and the blue lines $\left\{\mathcal{B}_{1}, \mathcal{B}_{2}, \ldots, \mathcal{B}_{e}\right\}$, we denote by $p_{i j}$ the intersection point $\mathcal{R}_{i} \cap \mathcal{B}_{j}$.

Let $\mathcal{A}$ be a subset of the nodes of a $(d \times e)$-cage, where $d \geq e$. We say that $\mathcal{A}$ is:

- triangular if, with respect to some ordering of the red and blue lines forming the cage, $\mathcal{A}$ is of the form $\left\{p_{i j}\right\}_{i+j \leq d}$;

- quasi-triangular if the cardinality of the nodes in $\mathcal{A}$ residing on a typical blue line ranges from $d$ to $d-e+1$ and takes each intermediate value exactly once;

- supra-triangular if, with respect to some ordering of the red and blue lines forming the cage, $\mathcal{A}$ is of the form $\left\{p_{i j}\right\}_{i+j \leq d+1}$; 
- supra-quasi-triangular if the cardinality of the set of nodes in $\mathcal{A}$ residing on a typical blue line ranges from $d$ to $d-e+2$, the value $d$ is achieved on two lines, and each value from $d-1$ to $d-e+2$ is achieved exactly once.
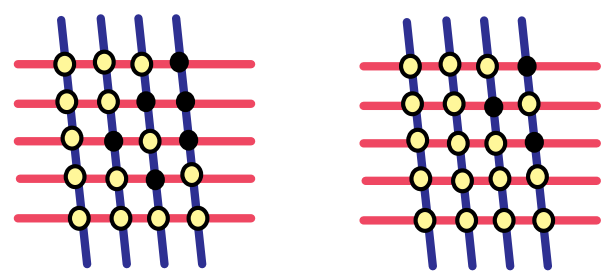

Figure 4 . A $(5 \times 4)$-cage with a quasi-triangular and an supraquasi-triangular sets of nodes.

We now formulate our generalization of the $(3 \times 3)$-cage theorem. Later, we will show how this result can be also derived with the aid of Bacharach duality (Theorem 4.1).

\section{Theorem 3.1. (Cage Theorem for Plane Curves).}

1. If a curve in $\mathbb{P}^{2}$ of degree $d$ passes through an supra-quasi-triangular set $\mathcal{A}$ of nodes of a $(d \times e)$-cage with $d \geq e$, then it passes through all the nodes of the cage.

2. No curve of degree less than e can pass through a quasi-triangular set of nodes of a $(d \times e)$-cage when $d \geq e$.

Remark. Not every subset of nodes with the cardinality of an supra-triangular set $\mathcal{A}$ will have the property claimed by Theorem 3.1. For example, for a $(4 \times 4)$ cage, $|\mathcal{A}|=13$. If $\mathcal{Y}$ is the complement of the set $\left\{p_{42}, p_{43}, p_{44}\right\}$ in the nodal set of a $(4 \times 4)$-cage, then not every curve $\mathcal{C}$ of degree four that contains $\mathcal{Y}$ passes through the three nodes $p_{42}, p_{43}$ and $p_{44}$ : just take $\mathcal{C}$ to be the union of the lines $\mathcal{B}_{1}, \mathcal{B}_{2}$, and $\mathcal{B}_{3}$ with a line $\mathcal{L}$ containing $p_{41}$ but missing $p_{42}, p_{43}$, and $p_{44}$. Therefore, the combinatorial structure of an supra-quasi-triangular set is important for the truth of Theorem 3.1. This example indicates the complexity of generalizing the $(4 \times 4)$ cage theorem to sets of nodes obtained by intersecting two generic curves of degree four.

Proof. We prove Theorem 3.1 for cages in $\mathbb{A}^{2}$. The argument for cages in $\mathbb{P}^{2}$ is similar. First, for statement (1) we order the blue lines so that the first blue line contains $d$ nodes of $\mathcal{A}$, the second blue line also contains $d$ nodes, the third blue line contains $d-1$ nodes, the fourth $d-2$ nodes, and so on, to the last line, which carries $d-e+2$ nodes (as depicted in Figure 4, the right-hand diagram).

Let $R_{i}$ be a linear polynomial in $x$ and $y$ having the line $\mathcal{R}_{i}$ as its zero set, let $B_{i}$ be a linear polynomial with the line $\mathcal{B}_{i}$ as its zero set, and let $R=\prod_{i=1}^{d} R_{i}$ and $B=\prod_{j=1}^{e} B_{j}$. Then any linear combination $S_{\lambda, \mu}:=\lambda R+\mu B$ vanishes at each node of the cage. Let $\mathcal{S}_{[\lambda: \mu]}$ be the curve of degree $d$ defined by the equation $S_{\lambda, \mu}=0$. This curve depends on the choice of the polynomials $R$ and $B$ representing the cage. However, the family $\left\{\mathcal{S}_{[\lambda: \mu]}\right\}$ of such curves is determined completely by the cage.

Let $P$ be any polynomial of degree at most $d$ that vanishes at the nodes from $\mathcal{A}$. We aim to show that, for appropriate numbers $\lambda$ and $\mu$ and some polynomial $Q, P$ is of the form $\lambda R+\mu B \cdot Q$, and therefore must vanish at every node. 
The argument is reductive in nature. Compare the restrictions of $P$ and of $S_{\lambda, \mu}$ to the blue line $\mathcal{B}_{1}$. Since both restrictions are just polynomials of degree at most $d$ in a single variable (say, the parameter $m$ in a parametric description of $\mathcal{B}_{1}$ ) that share a set of $d$ roots - namely, the nodes $p_{11}, p_{12}, \ldots, p_{1 d}$ - they must be proportional. By choosing an appropriate $\lambda=\lambda_{\star}$, we ensure that $P-S_{\lambda_{\star}, \mu}$ is identically zero along the line $\mathcal{R}_{1}$. Since $B_{1}$ is an irreducible polynomial, $P-S_{\lambda_{\star}, \mu}$ must be divisible by $B_{1}$. This statement follows from Hilbert's Nullstellensatz (see [10, Theorem 1.3A]). However, a more elementary argument also applies: just pick new local coordinates $\left(x^{\prime}, y^{\prime}\right)$ so that $x^{\prime}$ is given by the linear polynomial $B_{1}$. In the new coordinates, the polynomial $P-S_{\lambda_{\star}, \mu}$ transforms into a polynomial $T\left(x^{\prime}, y^{\prime}\right)$ in $x^{\prime}$ and $y^{\prime}$. Since $T\left(0, y^{\prime}\right)=0$ identically in $y^{\prime}$, all the monomials making up $T$ must be divisible by $x^{\prime}=B_{1}$. Therefore, $P=S_{\lambda_{\star}, \mu}+B_{1} \cdot P_{1}$, where $P_{1}$ is a polynomial of degree at most $d-1$. Because all the nodes of the set $\mathcal{A}$ are distinct, $P_{1}$ must vanish at the $d$ nodes of $A \cap \mathcal{B}_{2}$ (indeed, $B_{1}$ is nonzero on $A \cap \mathcal{B}_{2}$ ). As a consequence, the restriction of $P_{1}$ to the line $\mathcal{B}_{2}$ vanishes. Hence, $P_{1}$ is divisible by $B_{2}$. The original $P$ acquires the form $P=S_{\lambda_{\star}, \mu}+\left(B_{1} B_{2}\right) \cdot P_{2}$, where $P_{2}$ is a polynomial of degree at most $d-2$. This algorithm can be repeated again and again until we reach the conclusion that $P=S_{\lambda_{\star}, \mu}+\left(B_{1} B_{2} \ldots B_{e}\right) \cdot P_{e}$, where $P_{e}$ is of degree $d-e$. Hence $P$ must be of the form $\lambda_{\star} R+B \cdot Q$. In particular, when $e=d$, we obtain $P=S_{\lambda_{\star}, \mu_{\star}}$ for an appropriate $\mu_{\star}$.

The second statement of the cage theorem has an even simpler proof. Let $Q$ be a polynomial of degree less than $e$ that vanishes on a quasi-triangular set $T$ of nodes. Then, by an argument similar to the one just presented, its restrictions to any blue line must be identical zero: for an appropriate $P_{m}$, the number of roots on $\mathcal{B}_{m}$ exceeds its degree. Thus, $Q$ must be divisible by $B_{1} B_{2} \ldots B_{e}$, which is only possible when $Q$ is the zero polynomial.

Recall that curves of degree $d$ in $\mathbb{P}^{2}$ are parameterized by points of the projective space $\mathbb{P}_{*}^{\left(d^{2}+3 d\right) / 2}$ of dimension $\left(d^{2}+3 d\right) / 2$. The requirement that a curve of degree $d$ pass through a given point in $\mathbb{P}^{2}$ imposes a homogeneous linear constraint on the homogeneous coordinates in $\mathbb{P}_{*}^{\left(d^{2}+3 d\right) / 2}$. Because this constraint is homogeneous, any system of $\left(d^{2}+3 d\right) / 2$ such equations has a nontrivial solution. In other words, for any given $\left(d^{2}+3 d\right) / 2$ points in $\mathbb{P}^{2}$ there exists a curve of degree $d$ that contains them.

On the other hand, the cardinality of an supra-quasi-triangular set $\mathcal{A}$ of nodes in a $(d \times d)$-cage is

$$
d^{2}-[(d-1)(d-2) / 2]=\left[\left(d^{2}+3 d\right) / 2\right]-1,
$$

one less than the dimension of the space of degree $d$ curves! No wonder that the curves that pass through an supra-quasi-triangular set $\mathcal{A}$ of a $(d \times d)$-cage form (according to Theorem 3.2) a one-parameter family, a line $\mathbb{P}_{*}^{1}$ in $\mathbb{P}_{*}^{\left(d^{2}+3 d\right) / 2}$. The proof of the cage theorem tells us that all the $\left[\left(d^{2}+3 d\right) / 2\right]-1$ nodes from $\mathcal{A}$ impose independent linear restrictions: the nodes of $\mathcal{A}$ are generically located. Evidently, the rest of the nodes have extremely special locations. The requirement that a curve of degree $d$ go through the nodes of an supra-quasi-triangular set leaves only one degree of freedom. Furthermore, since any such curve is of the form $\mathcal{S}_{[\lambda: \mu]}$, the vanishing of the polynomial at any point in $\mathbb{P}^{2}$ distinct from the nodes of the cage fixes the proportionality class $[\lambda: \mu]$ and, with it, the corresponding curve 
in the family $\left\{\mathcal{S}_{[\lambda: \mu]}\right\}$. In particular, when $e=d$, any curve of degree less than $d$ containing an supra-quasi-triangular set $\mathcal{A}$ of nodes and a point on a red (blue) line distinct from the nodes is the union of red (blue) lines that form the cage.

What are some implications of Theorem 3.1? We have seen that any polynomial of degree at most $d$ that vanishes on an supra-quasi-triangular nodal set $\mathcal{A}$ of a $(d \times e)$-cage is of the form $\lambda R+B \cdot Q$, where $\operatorname{deg} Q \leq d-e$. Therefore, for any set $\mathcal{F}$ of $\left[(d-e)^{2}+3(d-e)\right] / 2$ points located on the red lines of the cage, there is a polynomial $Q$ that vanishes on $\mathcal{F}$. As a result, the zero set of $\lambda R+B \cdot Q$ contains all the nodes of the cage together with the set $\mathcal{F}$. For a generic set $\mathcal{F}$ such curves form a one-parameter family. This leads to Corollaries 3.1 and 3.2.

We say that a finite set $\mathcal{B}$ is a minimally redundant set for polynomials of degree $d$ if for some element $b$ of $\mathcal{B}$ vanishing on the set $\mathcal{B} \backslash\{b\}$ imposes $|\mathcal{B}|-1$ independent linear constraints on the coefficients of such polynomials and if any polynomial of degree $d$ that vanishes on $\mathcal{B} \backslash\{b\}$ automatically vanishes on $\mathcal{B}$.

Corollary 3.1. Vanishing requirements at the points of an supra-quasi-triangular set $\mathcal{A}$ of a $(d \times e)$-cage with $e \leq d$ impose independent conditions on polynomials of degrees at least $d$. Further, adding any new node to $\mathcal{A}$ produces a minimally redundant set $\mathcal{B}$ that fails to impose independent restrictions on polynomials of degree $d$.

Corollary 3.2. If $\mathcal{A}$ is an supra-quasi-triangular nodal set of $a(d \times e)$-cage $K$ and $\mathcal{F}$ is a set $\left[(d-e)^{2}+3(d-e)\right] / 2$ points located on the red lines of $K$, then there exists a one-parameter family of curves of degree $d$ that contain $\mathcal{F}$ and $\mathcal{A}$. Such curves pass through the rest of the nodes of $K$.

For a $(d \times d)$-cage $K$, an supra-quasi-triangular nodal set $\mathcal{A}$ of $K$, and a point $p$ different from the nodes of $K$ there exists a unique curve $\mathcal{C}$ of degree $d$ that passes through $p$ and $\mathcal{A}$. The curve $\mathcal{C}$ contains all the nodes of $K$.

Since at each node $p_{i j}$ of a cage $K$ the red and blue lines $\mathcal{R}_{i}$ and $\mathcal{B}_{j}$ are transversal and since no other lines of the cage pass through $p_{i j}$, one can show that any curve $\mathcal{S}_{[\lambda: \mu]}$ is nonsingular at the nodes. It suffices to check that for any $\lambda$ and $\mu$ the gradient of $S_{\lambda, \mu}$ is nonzero at $p_{i j}$. The argument is based on the product rule for gradients. The gradient $\nabla S_{\lambda, \mu}$ of $S_{\lambda, \mu}$ at $p_{i j}$ is given by

$$
\nabla S_{\lambda, \mu}\left(p_{i j}\right)=\lambda r_{i j} \cdot \nabla R_{i}\left(p_{i j}\right)+\mu b_{i j} \cdot \nabla B_{j}\left(p_{i j}\right),
$$

where $r_{i j}=\prod_{k \neq i} R_{k}\left(p_{i j}\right)$ and $b_{i j}=\prod_{k \neq j} B_{k}\left(p_{i j}\right)$ are nonzero. Thus the nontrivial linear combination of independent vectors $\nabla R_{i}\left(p_{i j}\right)$ and $\nabla B_{j}\left(p_{i j}\right)$ is a nonzero vector. This implies that each curve $\mathcal{S}_{[\lambda: \mu]}$ has a well-defined tangent line at each of the nodes. Further, as (1) testifies, in the cage family there is a unique curve tangent to a given line $\tau_{i j}^{*}$ in $\mathbb{P}_{*}^{2}$ that pass through a given node $p_{i j}$. Indeed, one can reconstruct $[\lambda: \mu]$ from the three vectors $r_{i j} \cdot \nabla R_{1}\left(p_{i j}\right), b_{i j} \cdot \nabla B_{1}\left(p_{i j}\right)$, and $\nabla S_{\lambda, \mu}\left(p_{i j}\right)$.

In this way we can define a directional line field $\tau(\lambda, \mu)$ at the nodes of the cage, the one generated by the tangent lines to the curve $\mathcal{S}_{[\lambda: \mu]}$. Each direction, say $\tau_{11}(\lambda, \mu)$, determines the rest, so that the field $\tau(\lambda, \mu)$ is "rigid." This helps to establish the following result:

Theorem 3.2. If $K$ is a $(d \times d)$-cage in $\mathbb{P}^{2}$ and $\tau_{i j}$ in $\mathbb{P}_{*}^{2}$ is a direction at one of its nodes $p_{i j}$, then there exists a unique curve $\mathcal{S}_{[\lambda: \mu]}$ of degree $d$ that passes through all the nodes of $K$ and has $\tau_{i j}$ as its tangential direction at the point $p_{i j}$. 
Moreover, the same conclusion holds for any curve of degree $d$ passing through an supra-quasi-triangular nodal set $\mathcal{A}$ of $K$ and having $\tau_{i j}$ as the tangential direction at a given point $p_{i j}$ of $\mathcal{A}$.

We are now in a position to derive a nice generalization (Theorem 3.3) of the Pascal theorem. It is based simply on Theorem 3.1 and counting dimensions.

The variety of $(d \times d)$-cages is $4 d$-dimensional: each such cage is determined by a configuration of $d$ red and $d$ blue points in $\mathbb{P}_{*}^{2}$. In fact, a $(d \times d)$-cage $K$ can be reconstructed from certain sets consisting of $2 d$ nodes. Not any $2 d$ nodes will do, for in order to determine the cage, they must satisfy certain combinatorial conditions. One such set $\mathcal{D}$ consists of the nodes $p_{i j}$ subject to the constraints $i+j=d \pm 1$ together with two "corners" $p_{d 1}$ and $p_{1 d}$ (see Figure 5 ). Note that $\mathcal{D}$ is contained in

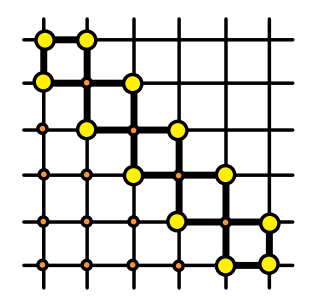

FiguRe 5.

$\mathcal{A}=\left\{p_{i j}\right\}_{i+j \leq d+1}$, which is an supra-triangular set. The complementary set $\mathcal{A} \backslash \mathcal{D}$ consists of

$$
\left\{\left[\left(d^{2}+3 d\right) / 2\right]-1\right\}-2 d=[d(d-1) / 2]-1
$$

elements. In fact, any subset $\mathcal{D}$ of $\mathcal{A}$ with the property that any line from the cage hits it at exactly two points would be a good choice.

Assume for the moment that all $2 d$ nodes from the set $\mathcal{D}$ are located on an irreducible curve $\mathcal{Q}$ of degree $u$ less than $d$. We wish to determine when the nodes from the set $\mathcal{A} \backslash \mathcal{D}$ are located on a curve $\mathcal{Q}_{\star}$ of complementary degree $v=d-u$. Since there are $[d(d-1) / 2]-1$ elements in $\mathcal{A} \backslash \mathcal{D}$, the existence of such a curve is guaranteed by the inequalities

$$
\left[\left(v^{2}+3 v\right) / 2\right] \geq[d(d-1) / 2]-1
$$

and $1 \leq v \leq d-1$. Remarkably, when $d$ is at least three, $u=2$ and $v=d-2$ satisfy these conditions,

$$
\left[(d-2)^{2}+3(d-2)\right] / 2=[d(d-1) / 2]-1,
$$

whereas any choice of $u$ and $v$ with $u>2$ fails to obey them! Therefore, the points of $\mathcal{A} \backslash \mathcal{D}$ are located on a curve $\mathcal{Q}_{\star}$ of degree $d-2$.

Consider the reducible curve $\mathcal{C}=\mathcal{Q} \cup \mathcal{Q}_{\star}$ of degree $d$ with an irreducible quadratic component $\mathcal{Q}$. The nodes of $\mathcal{D}$ are located on $\mathcal{Q}$, and the nodes from $\mathcal{A} \backslash \mathcal{D}$ are on $\mathcal{Q}_{\star}$. Thus, all the nodes from $\mathcal{A}$ are located on $\mathcal{C}$. By Theorem 3.1, all the nodes of the cage $K$ must lie on $\mathcal{C}$ as well. However, the nodes of $K$ that are not in $\mathcal{D}$ cannot belong to $\mathcal{Q}$, because this would contradict Bezout's theorem (a line would hit the irreducible quadratic curve $Q$ at three points). It follows that the unaccounted for nodes must be located on $\mathcal{Q}_{\star}$. 
We arrive in this way at our main geometric result - a generalized Pascal's theorem. The $2 d$-gram that depicts it is still a bit mysterious, so we retain Pascal's description.

Theorem 3.3. (The Mystic 2d-Gram). Let $\mathcal{D}$ be a polygon with $2 d$ sides, colored in two alternating colors and inscribed in a quadratic curve $\mathcal{Q}$. If $K$ is the $(d \times d)$-cage generated by $\mathcal{D},{ }^{3}$ then all $\left(d^{2}-2 d\right)$ new nodes of the cage lie on a plane curve $\mathcal{Q}_{\star}$ of degree at most $d-2$. For a generic polygon $\mathcal{D}$, the curve $\mathcal{Q}_{\star}$ is unique.

Figures 6 and 7 show the mystic octagram and decagram, respectively, that generate quadratic and cubic curves $\mathcal{Q}_{\star}$. We remark that the $2 d$-gon $\mathcal{D}$ in Theorem

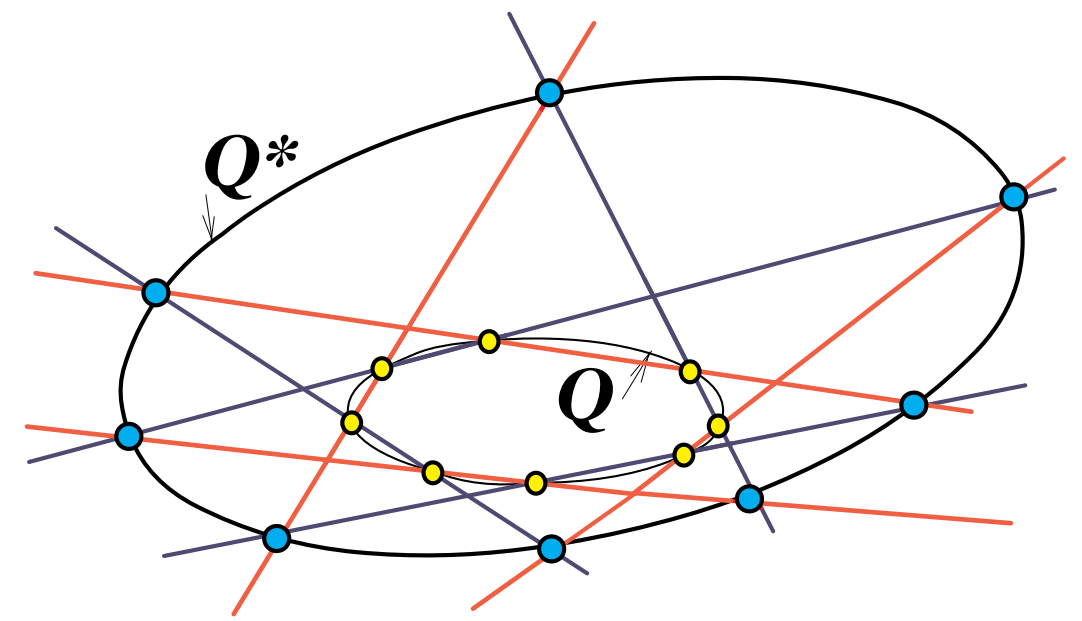

FiguRE 6. Mystic octagram.

3.3 can be a union of two or more $2 k$-gons $(1<k<d)$, each of which is bicolored in an alternating manner. Also, as two vertices merge, some of the sides of the $2 d$-gon can become tangent to the quadratic curve. In particular, if all the red lines become tangent, one obtains a degenerate cage formed by a red circumscribed $d$-gon and a blue inscribed one, the vertices of the blue polygon being the points of tangency for the red one. Extending Theorem 3.3 by continuity, the rest of the nodes of the resulting cage must lie on a curve of degree $d-2$.

Contemplating Theorem 3.3, one might wonder: Which curves $\mathcal{Q}_{\star}$ of degree $d-2$ can be produced via the mystic $2 d$-gon construction from a $2 d$-gon inscribed in a (given) quadratic $\mathcal{Q}$ ? Clearly, for large $d$ such curves $\mathcal{Q}_{\star}$ will be exceptional. However, for a few small $d$ we might have a chance to manufacture almost any plane curve of degree $d-2$ as a $\mathcal{Q}_{\star}$. A count of dimensions provides a crude indication to the possible degrees $d$ for which this might be true. For $d=3$ the space of hexagons inscribed in a particular $\mathcal{Q}$ is six-dimensional, and the space of lines in $\mathbb{A}^{2}$ is twodimensional. For $d=4$ the space of octagons inscribed in $\mathcal{Q}$ is eight-dimensional, while the space of quadratic curves is five-dimensional. For $d=5$ the space of

\footnotetext{
${ }^{3}$ The vertices of $\mathcal{D}$ are among the nodes of $K$.
} 


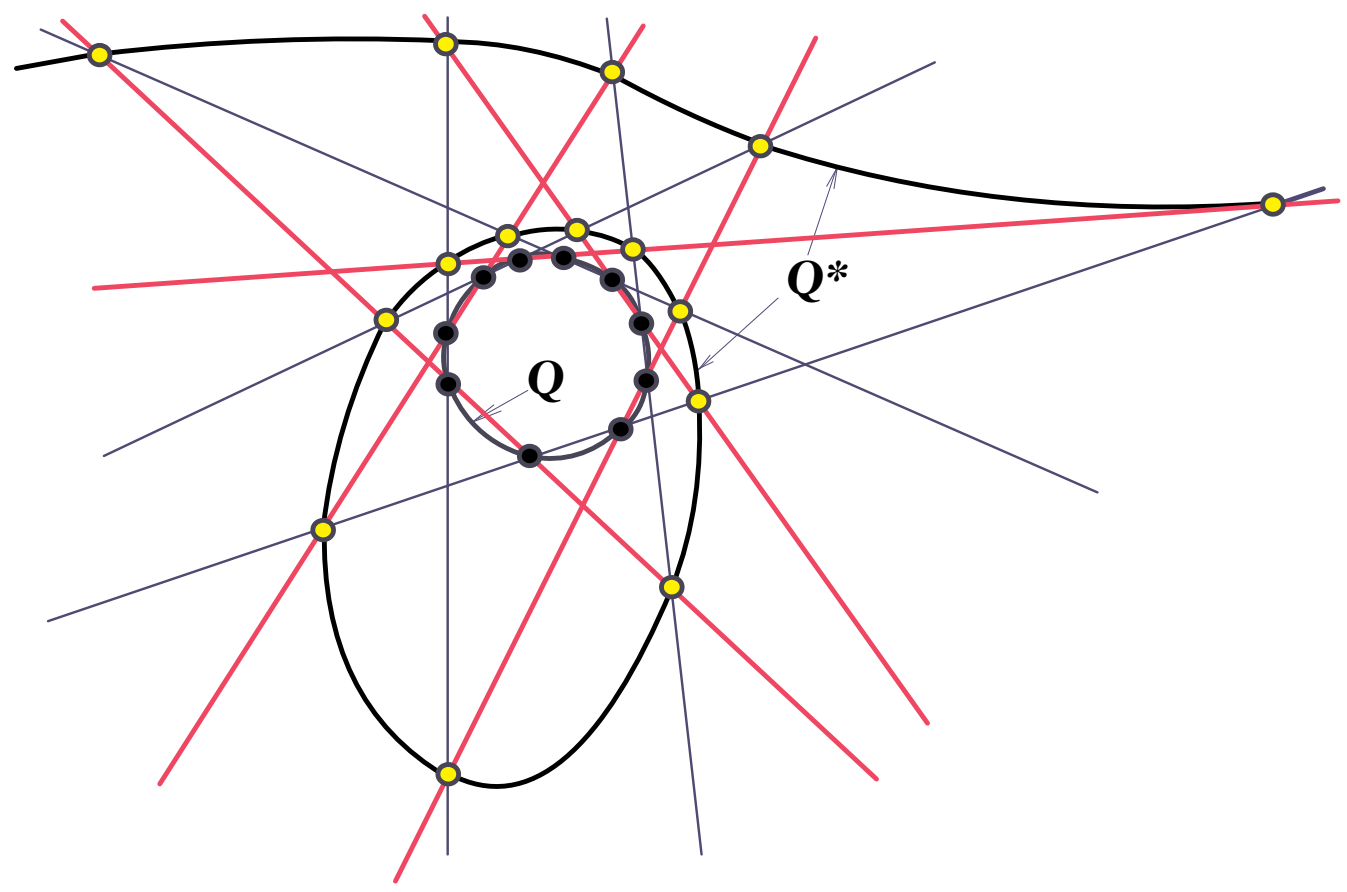

Figure 7. Mystic decagram.

decagons inscribed in in $\mathcal{Q}$ is ten-dimensional, and the space of cubics is ninedimensional. So far, so good! But already for $d=6$ the dimension of the inscribed 12-gons is twelve, whereas the dimension of quartics is fourteen. Accordingly, not every quartic can be a $\mathcal{Q}_{\star}$ for a fixed $\mathcal{Q}$. However, if we allow ourselves to vary the quadratic curve $\mathcal{Q}$ as well, we gain five extra degrees of freedom. This takes us through the next case $d=7$ : the space of quintics is 19-dimensional, and $19=14+5$. Once $d$ exceeds seven, the $\mathcal{Q}_{\star}$ s form a subvariety in the space of curves of degree $d-2$. We conjecture that any plane algebraic curve of degree seven or less can be obtained from some $2 d$-gon inscribed in some quadratic curve via the corresponding $2 d$-gram.

The next corollary reflects an interesting duality in the universe of quadratic curves marked with eight ordered points (see Figure 6).

Corollary 3.3. (Mystic Octagram Duality). Let $\mathcal{D}$ be a bicolored octagon inscribed in a quadratic curve $\mathcal{Q}$. If $K$ is the $(4 \times 4)$-cage generated by $\mathcal{D}$, then the eight new nodes of the cage lie on a quadratic curve $\mathcal{Q}_{\star}$ and give rise to a new bicolored octagon. For a generic inscribed octagon $\mathcal{D}$, the curve $\mathcal{Q}_{\star}$ is unique.

In particular, the duality tells us that any quadratic curve $\mathcal{Q}$ can be generated from another quadratic curve $\mathcal{Q}_{\star}$ via the mystic octagram construction.

The octagon in the corollary can be a union of two (bicolored) quadrilaterals. It can also degenerate into a union of two (monocolored) quadrilaterals, one of which is tangent to the quadratic curve and the other of which has its vertices at the points of tangency. 
Curiously, quadratic curves play a special role in the mystic $2 d$-gram. As our count of dimensions shows, in general, nothing can be claimed about $2 d$-gons inscribed in curves of degree $u$ when $2<u<d$. No valid Pascal's theorems inhabit that range! Nevertheless, the situation is better than one might think, if one is willing to abandon the inscribed $2 d$-gons in favor of more elaborate inscribed subcages. But that is a subject for another day.

The following proposition is a well-known consequence of Bezout's theorem (see [11, Proposition 3.14]):

Theorem 3.4. If two curves $\mathcal{D}$ and $\mathcal{E}$ in $\mathbb{P}^{2}$ of degree $d$ intersect in exactly $d^{2}$ points and if exactly $k \cdot d$ of these points lie on an irreducible curve $\mathcal{Q}$ of degree $k$ with $k<d$, then the remaining $d(d-k)$ intersection points lie on a curve $\mathcal{Q}_{\star}$ of degree at most $d-k$.

We concentrate on a special case of Theorem 3.4-namely, the case where $\mathcal{D}$ and $\mathcal{E}$ are the red and blue lines of a $(d \times d)$-cage $K$. Each time we are able to produce a curve $\mathcal{Q}$ as in Theorem 3.4, a Pascal-type theorem will be revealed. However, the unresolved issue raised by Theorem 3.4 is how to recognize curves $\mathcal{D}$ and $\mathcal{E}$ whose intersection set admits an irreducible curve $\mathcal{Q}$ of degree $k$ passing through $k \cdot d$ nodes. We do not have a satisfactory answer even in the case when $\mathcal{D}$ and $\mathcal{E}$ are each unions of $d$ lines. This seems to be a challenging problem. To appreciate the challenge, consider a $(d \times d)$-cage in $\mathbb{A}^{2}$ whose nodes have integral coordinates, whose red lines are among the horizontal lines forming the integral grid, and whose blue lines are among the vertical lines of this grid. Such a cage is characterized by two unordered $d$-tuples of integers $\left\{n_{i}\right\}_{1 \leq i \leq d}$ and $\left\{m_{j}\right\}_{1 \leq j \leq d}$, the $x$ - and $y$-intercepts of the blue and red lines, respectively.

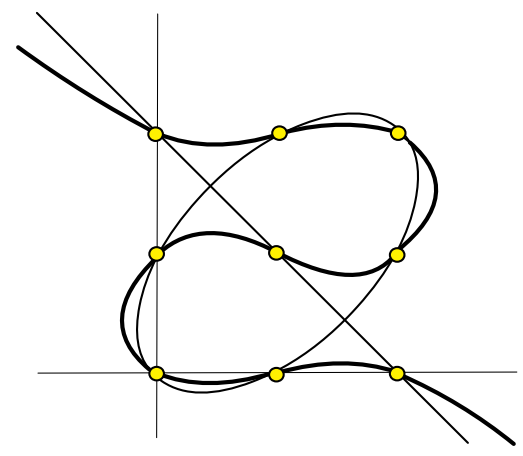

FiguRE 8. A cubic degenerating into a line and a conic.

Problem. Describe in terms of $\left\{n_{i}\right\}$ and $\left\{m_{j}\right\}$ the $(d \times d)$-cages that admit reducible curves of degree $d$ passing through all their nodes other than the $\mathcal{D}$ and $\mathcal{E}$ forming the cage. Specifically, we ask: Which of these cages admit irreducible curves $\mathcal{Q}$ of degree $k(<d)$ that contain $k \cdot d$ nodes?

One class of cages with the desired property is easy to produce. These are the cages with collinear "diagonal" (with respect to some ordering) nodes $p_{i i}$. Figures 8 and 9 display them, together with the Pascal-type diagrams that they support. The figures also document how an irreducible curve attached to the nodes of such a cage can degenerate into a reducible one. 


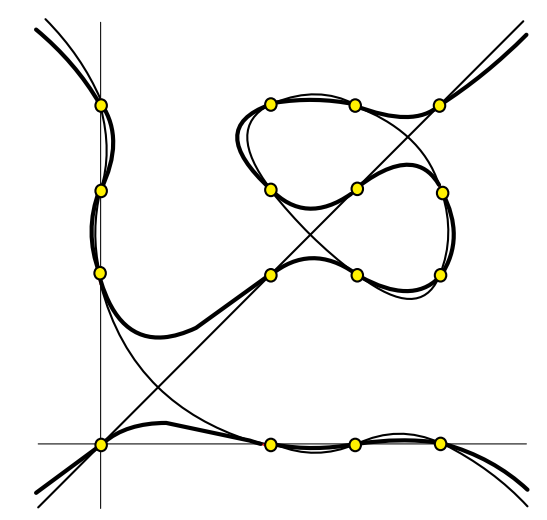

Figure 9. A quartic degenerating into a line and a cubic.

Theorem 3.4 coupled with the cage theorem implies yet another generalization of Pascal's theorem:

Corollary 3.4. The nondiagonal nodes of a $(d \times d)$-cage lie on a curve $\mathcal{Q}$ of degree $d-1$ if and only if the $d$ diagonal nodes of the cage are collinear.

\section{CAGES AND MODERNITY}

We close this article by incorporating the elementary considerations of previous sections into the language of classical and contemporary algebraic geometry. Denote by $\mathcal{V}_{k}$ the vector space of homogeneous polynomials of degree $k$ in $x, y$, and $z$. Its dimension is $(k+1)(k+2) / 2$. Let $\mathcal{X}$ be an algebraic subset of $\mathbb{P}^{2}$, that is, the solution set for a system of equations in the variables $x, y$, and $z$ defined by homogeneous polynomials. We denote by $I_{\mathcal{X}}$ the ideal of the polynomial ring $\mathbb{C}[x, y, z]$ generated by polynomials that vanish on $\mathcal{X}$. The vector subspace $I_{\mathcal{X}, k}$ of $I_{\mathcal{X}}$ comprises its homogeneous polynomials of degree $k$.

The Hilbert function $h_{\mathcal{X}}: \mathbb{Z}_{+} \rightarrow \mathbb{Z}_{+}$of an algebraic set $\mathcal{X}$ is defined by the formula $h_{\mathcal{X}}(k)=\operatorname{dim}\left(\mathcal{V}_{k}\right)-\operatorname{dim}\left(I_{\mathcal{X}, k}\right)$, where "dim" signifies the dimension of a vector space. For sufficiently large $k, h_{\mathcal{X}}(k)$ behaves like a polynomial in $k$ of degree $\operatorname{dim}(\mathcal{X})$, the complex "topological" dimension. In fact, if $\mathcal{X}$ is a zero-dimensional set, then for all sufficiently large $k, h_{\mathcal{X}}(k)=|\mathcal{X}|$, the cardinality of $\mathcal{X}$. When $\mathcal{X}$ is a curve, $h_{\mathcal{X}}(k)$ is asymptotically a linear polynomial of the form $\operatorname{deg}(\mathcal{X}) \cdot k+c$, where $c$ is a constant (see [10, Theorem 7.5]).

The following important theorem describes an intricate duality relation between the Hilbert function of a complete intersection (roughly speaking, complete intersections are algebraic sets whose codimension in the ambient projective space is equal to the number of polynomial equations that define them) and the Hilbert functions of its complementary parts [1] (see [5, Theorem CB5] and [4] for significant generalizations):

Theorem 4.1. (Bacharach). Let $\mathcal{D}$ and $\mathcal{E}$ be projective plane curves of degrees $d$ and e, respectively, and let the finite set $\mathcal{X}=\mathcal{D} \cap \mathcal{E}$ be a complete intersection. Assume that $\mathcal{X}$ is the disjoint union of two subsets $\mathcal{X}_{1}$ and $\mathcal{X}_{2}$. Then for any $k$ 
satisfying $k \leq d+e-3$

$$
h_{\mathcal{X}}(k)-h_{\mathcal{X}_{1}}(k)=\left|\mathcal{X}_{2}\right|-h_{\mathcal{X}_{2}}((d+e-3)-k) .
$$

The left-hand side of formula (2) can be viewed as $\operatorname{dim}\left(I_{\mathcal{X}_{1}, k} / I_{\mathcal{X}, k}\right)$, while the right-hand side measures the failure of $\mathcal{X}_{2}$ to impose independent conditions on the polynomials of the "complementary" degree $(d+e-3)-k$. Substituting $d$ for $k$ in formula (2), we conclude that $h_{\mathcal{X}}(d)=h_{\mathcal{X}_{1}}(d)$ if and only if $\left|\mathcal{X}_{2}\right|=h_{\mathcal{X}_{2}}(e-3)$. In other words, the following two properties are equivalent: 1) any curve of degree $d$ that contains $\mathcal{X}_{1}$ must contain $\mathcal{X}$ as well, and 2) $\left|\mathcal{X}_{2}\right|=h_{\mathcal{X}_{2}}(e-3)$. For instance, with $d=e=3$, we get the Chasles and $(3 \times 3)$-cage theorems; if $\left|\mathcal{X}_{2}\right|=1$, then $h_{\mathcal{X}_{2}}(3-3)=1$ (a polynomial of degree zero that vanishes at the singleton $\mathcal{X}_{2}$ must be zero), which by the Bacharach theorem implies that $h_{\mathcal{X}}(3)=h_{\mathcal{X}_{1}}(3)$. In other words, $I_{\mathcal{X}, 3}=I_{\mathcal{X}_{1}, 3}$, so any cubic polynomial that vanishes on $\mathcal{X}_{1}$ of cardinality eight vanishes on $\mathcal{X}$ of cardinality nine.

We now see how Theorem 3.1 can be derived from Theorem 4.1. To simplify the combinatorics, we consider only $(d \times d)$-cages and only triangular and supratriangular sets of nodes. We work by induction on $d$. Assume that the cage theorem (which consists of two claims) is true whenever $d<d_{\star}$. In particular, this inductive assumption implies that, when $d<d_{\star}$, any polynomial of degree less than $d$ that vanishes at the points of a triangular set in a $(d \times d)$-cage is identically zero.

Let $\mathcal{A}$ denote an supra-triangular set of nodes in a $\left(d_{\star} \times d_{\star}\right)$-cage, and let $\mathcal{B}$ be its complementary set. The first statement of the cage theorem can be expressed as $\mathcal{I}_{\mathcal{A}, d_{\star}}=\mathcal{I}_{\mathcal{A} \cup \mathcal{B}, d_{\star}}$ which is equivalent to the claim that $h_{\mathcal{A}}\left(d_{\star}\right)=h_{\mathcal{A} \cup \mathcal{B}}\left(d_{\star}\right)$. By Theorem 4.1, this is equivalent to the property $h_{\mathcal{B}}\left(d_{\star}-3\right)=|\mathcal{B}|$. Note that $\mathcal{B}$ is a triangular set for an appropriate $\left(d_{\star}-2\right) \times\left(d_{\star}-2\right)$-subcage. By the inductive assumption, any polynomial of degree $d_{\star}-3$ that vanishes on $\mathcal{B}$ is the zero polynomial, that is, $\mathcal{I}_{\mathcal{B}, d_{\star}-3}=\{0\}$. Hence $h_{\mathcal{B}}\left(d_{\star}-3\right)=\operatorname{dim}\left(\mathcal{V}_{d_{\star}-3}\right)$. On the other hand, $\operatorname{dim}\left(\mathcal{V}_{d_{\star}-3}\right)=\left(d_{\star}-1\right)\left(d_{\star}-2\right) / 2=|\mathcal{B}|$, which completes the inductive step for the first statement of the cage theorem.

The inductive treatment of the second statement is similar to the one presented in Theorem 3.1. Consider a polynomial $P$ of degree $d_{\star}-1$ that vanishes on a triangular set $\mathcal{T}$ of a $\left(d_{\star} \times d_{\star}\right)$-cage. Then it vanishes at the $d_{\star}$ nodes of the cage that lie on the line $\mathcal{B}_{1}: B_{1}=0$. As a result, $P$ must be divisible by $B_{1}$. Let $P=B_{1} \cdot Q$. Then $Q$ is of degree $d_{\star}-2$ and must vanish at the nodes of $\mathcal{T}$ that do not belong to $\mathcal{B}_{1}$. They form a triangular set of a $\left(\left(d_{\star}-1\right) \times\left(d_{\star}-1\right)\right)$-cage. Thus, by induction, $Q$ is the zero polynomial, and so must be $P$.

Although in our investigations we have managed without Hilbert functions, they are invaluable in modern research relevant to the subject of our paper (see [6], [7], $[\mathbf{8}],[\mathbf{9}])$. The interested reader may examine $[\mathbf{9}$, Theorem 3.13] to get a feel for the most recent developments in the field. This comprehensive result by Geramita, Harita, and Shin is a Fubini-type theorem for the Hilbert function of a finite subset $\mathcal{X}$ of $\mathbb{P}^{n}$ that is contained in the union of a family of hypersurfaces $\left\{\mathcal{V}_{i}\right\}_{1 \leq i \leq s}$ whose degrees $d_{i}$ add up to the degree of $\mathcal{X}$. Under a subtle hypothesis that regulates the interaction between $\mathcal{X}$ and the hypersurfaces, a nice formula emerges:

$$
h_{\mathcal{X}}(k)=h_{\mathcal{X} \cap \mathcal{V}_{1}}(k)+h_{\mathcal{X} \cap \mathcal{V}_{2}}\left(k-d_{1}\right)+\cdots+h_{\mathcal{X} \cap \mathcal{V}_{s}}\left(k-\left(d_{1}+\cdots+d_{s-1}\right)\right) .
$$

This formula could be instrumental in generalizing our results to multidimensional cages, or even to $\mathcal{X}_{\mathrm{s}}$ that are not necessarily complete intersections. 
ACKNOWLEDGMENTS. I am grateful to the referee, whose suggestions helped to improve the quality and scope of the original manuscript. In particular, his remarks helped me to clarify the relation between the cage theorem and its generalizations at the very center of more advanced and active research. My gratitude also goes to my colleagues Harry Tamvakis and Glen Van Brummelen, who helped me achieve a better exposition.

\section{REFERENCES}

[1] I. Bacharach, Über den Cayley'schen Schnittpunktsatz, Math. Ann. 26 (1886) 275-299.

[2] A. Cayley, On the Intersection of Curves, serialized in Cambridge Math. J., 25 - 27, and published by Cambridge University Press, Cambridge, 1889.

[3] M. Chasles, Traite de sections conique, Gauthier-Villars, Paris, 1885.

[4] E. D. Davis, A.V. Geramita, and F. Orecchia, Gorenstein algebras and Cayley-Bacharach theorem, Proceedings Amer. Math. Soc. 93 (1985) 593 - 597.

[5] D. Eisenbud, M. Green, and J. Harris, Cayley-Bacharach theorems and conjectures, Bull. Amer. Math. Soc. 33 (1996) 295-324.

[6] A. V. Geramita, and Y.S. Shin, $k$-configurations in $\mathbb{P}^{3}$ all have extremal resolutions, $J$. Algebra 213 (1999) 351- 368.

[7] A.V. Geramita, T. Harima, and Y.S. Shin, Extremal point sets and Gorenstein ideals, Adv. Math 152 (2000) $78-119$.

[8] - , An alternative to the Hilbert function for the ideal of a finite set of points in $\mathbb{P}^{n}$, Illinois J. 45 (2001) 1 - 23.

[9] - , Decompositions of the Hilbert Function of a Set of Points in $\mathbb{P}^{n}$, Canad. J. Math. 53 (2001) $925-943$.

[10] R. Hartshorne, Algebraic Geometry, Springer-Verlag, New York, 1977.

[11] M. Kirwan, Complex Algebraic Curves, Cambridge University Press, Cambridge, 1992.

[12] M. Ried, Undergraduate Algebraic Geometry, Cambridge University Press, Cambridge, 1998.

[13] N.B. Vasiliev, Pascal's hexagrams and cubic curves, Quant 8 (1987) 2 - 8 (Russian).

GABRIEL KATZ grew up in Kishinev, Moldova. He received his M.A. and Ph.D. degrees from Moscow State University. After immigrating to Israel in 1979, he taught at Tel Aviv University and later at Ben Gurion University, where he held a tenured position. In the early '90s, he moved with his family to the United States and settled down in the Boston area. Since then, he has held a variety of teaching and research positions at a number of universities and colleges: Rutgers, Brandeis, Harvard, MIT, Clark, Wellesley, and Bennington, to name a few. Currently, he is a research affiliate at Brandeis University. In the first half of his academic carrier, Dr. Katz studied symmetries of smooth manifolds. Lately, his work has acquired a more geometrical flavor; his articles include topics that cover: the topology of harmonic one-forms, three-dimensional topology, algebraic geometry of discriminant varieties, and more recently, the Morse Theory on manifolds with boundary. Dr. Katz also does research in the field of Mathematics Education.

Brandeis University, Waltham, MA 02454-9110

E-mail address:

gabrielkatz@rcn.com 